\title{
A common variant in 11q23.3 associated with hyperlipidemia is mediated by the binding and regulation of GATA4
}

\author{
Wen-Cheng Chou (iD) ${ }^{1}$, Wei-Ting Chen (D) $^{1}$ and Chen-Yang Shen $\mathbb{D}^{1,2} \bowtie$
}

\begin{abstract}
Large-scale genome-wide associations comprising multiple studies have identified hundreds of genetic loci commonly associated with hyperlipidemia-related phenotypes. However, single large cohort remains necessary in aiming to investigate ethnicity-specific genetic risks and mechanical insights. A community-based cohort comprising 23,988 samples that included both genotype and biochemical information was assembled for the genome-wide association analysis (GWAS) of hyperlipidemia. The analysis identified fifty genetic variants $\left(P<5 \times 10^{-8}\right)$ on five different chromosomes, and a subsequent validation analysis confirmed the significance of the lead variants. Integrated analysis combined with cell-based experiments of the most statistically significant locus in $11 q 23.3$ revealed rs651821 $\left(P=4.52 \times 10^{-76}\right)$ as the functional variant. We showed transcription factor GATA4 preferentially binds the T allele of rs651821, the protective allele for hyperlipidemia, which promoted APOA5 expression in liver cells and individuals with the $\Pi$ genotype of rs651821. As GATA4-APOA5 axis maintains triglyceride homeostasis, GATA4 activation by phenylephrine implies synergism for lowering triglyceride levels in hyperlipidemia patients. Our study demonstrates that rs651821 mediates APOA5 activation via allele-specific regulation by GATA4. We suggest elevating GATA4 activity could provide a therapeutic potential for treating the development of hyperlipidemia.
\end{abstract}

npj Genomic Medicine (2022)7:4 ; https://doi.org/10.1038/s41525-021-00279-5

\section{INTRODUCTION}

Dysregulation of plasma lipid levels contributes substantially to the development of several metabolic disorders, including cardiovascular diseases and type 2 diabetes mellitus ${ }^{1-4}$. Hyperlipidemia is primarily diagnosed by detecting elevated levels of triglycerides (TG) and/or total cholesterol (TC) in plasma samples ${ }^{5}$. Hyperlipidemia is a complex polygenic trait, and TG and TC levels in plasma are considered to be highly heritable ${ }^{6,7}$. In recent decades, genome-wide association studies (GWAS) have identified hundreds of loci that are associated with altered levels of TG, TC, low-density lipoprotein cholesterol (LDL-C), or high-density lipoprotein cholesterol $(\mathrm{HDL}-\mathrm{C})^{7,8}$. Most of these loci were initially identified in studies of populations of European ancestry (EUR) and later validated and confirmed in studies of East Asian populations (EAS) ${ }^{9}$. Genetic variants found with top-ranked significance are quite consistent between analyses of European and East Asian ancestors ${ }^{9}$. These include TC-associated loci in genes encoding the apolipoproteins $A P O E / C 1 / C 2$ (rs7412) and SORT1 (rs599839/rs629301) and TG-associated loci in APOA1/C3/ A4/A5 (rs662799/rs964184) and GCKR (rs1260326) genes. However, comparisons of loci among different ethnic groups have often shown that the magnitude of effect of each genetic variation can differ between populations. For example, in a multicenter study regarding EAS, researchers identified four new variants that were significantly $\left(P<5 \times 10^{-8}\right)$ associated with the levels of TG (rs10886863) and HDL-C (rs10743940, rs10504476 and rs 10144765$)^{9}$, yet none of these variants was significant $(P>$ $0.13)$ for $E U R^{8}$. These findings underscore the importance of considering ethnicity and population aspects when evaluating the significance of loci associated with the same metabolic traits.

Given that $88-94 \%$ of genetic variants identified from GWAS reside in non-coding regions, any effects on gene expression and disease phenotypes are expected to be mediated via regulation of promoters or enhancers ${ }^{10,11}$. Equally important, a growing body of evidence has shown that the lead single nucleotide polymorphisms (SNPs) identified by GWAS are rarely the causal variants that determine the traits $^{12,13}$. Consequently, the identification of true causal variants is critical for translating GWAS findings to disease prevention/intervention as well as for understanding the mechanism/etiology of diseases. Nevertheless, only a small portion of variants, i.e., among the $>100$ hyperlipidemia-associated loci identified by GWAS, have been elucidated with respect to their impact on the regulation of the lipid metabolism factors SORT1 and GCKR as well as VLDLR and ELOVL2 ${ }^{14-17}$. This result clearly shows that the biological/pathological contributions of the majority of GWAS-significant variants remain to be resolved.

In the present study, we analyzed hyperlipidemia-related genetic risk loci based on study participants from the Taiwan Biobank (TWB), which is the single largest cohort comprised of 23,988 individuals in $\operatorname{Taiwan}^{18}$. To define the pathological contribution of genetic variants, particular attention was given to the top-ranked locus 11q23.3, which revealed variant rs651821 as the causal variant affecting TG level, rather than variant rs662799 that has been repeatedly reported in several studies ${ }^{3,8,9}$. Additional functional experiments revealed that the transcription factor GATA4 binds specifically to the protective allele of rs651821 to modulate $A P O A 5$ expression and TG level. Consistently, similar result was observed through our in vivo analysis of clinical samples. We conclude that a genetic and molecular linkage exists between GATA4 and APOA5 via rs651821, which contributes to the maintenance of TG homeostasis.

\section{RESULTS}

\section{Identification of hyperlipidemia-associated genetic loci}

At the discovery stage of the GWAS analysis, 50 statistically significant SNPs $\left(P<5 \times 10^{-8}\right)$ were identified on chromosomes 2p23.3, 5q33.3, 8q24.13, 11q23.3, and 19q13.32 (see Fig. 1 for

\footnotetext{
${ }^{1}$ Institute of Biomedical Sciences, Academia Sinica, Taipei, Taiwan. ${ }^{2}$ College of Public Health, China Medical University, Taichung, Taiwan. ${ }^{凶}$ email: bmcys@ibms.sinica.edu.tw
} 


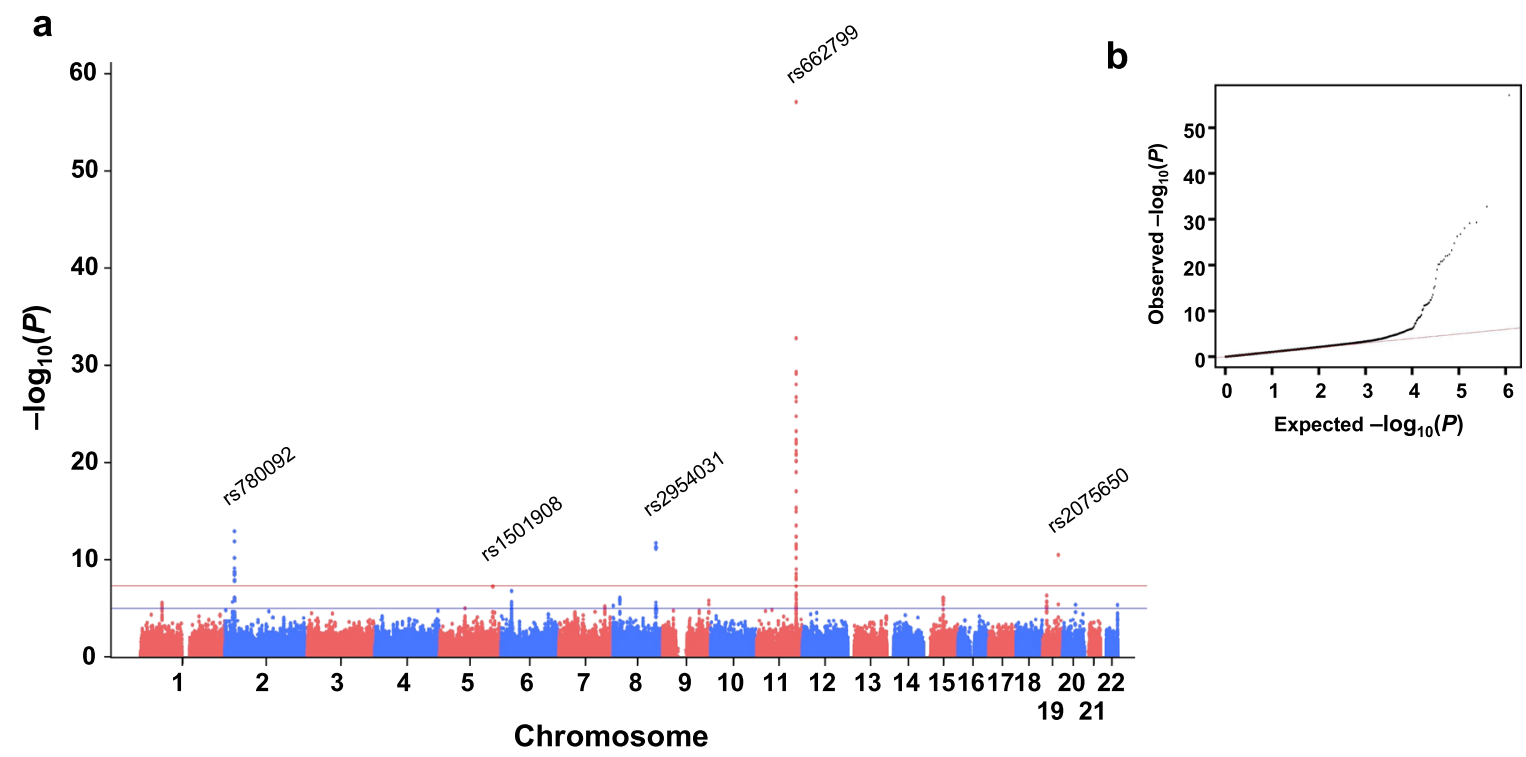

Fig. 1 Genome-wide analysis for hyperlipidemia. a Manhattan plot of results for the association between SNPs and hyperlipidemia risk. b Q-Q plot. The two horizontal lines in the Manhattan plot represent $P=1 \times 10^{-5}$ (lower) and $5 \times 10^{-8}$ (upper). The estimated inflation factor ( $\lambda$ ) was 1.019 for the GWAS.

Table 1. Top five lead variants identified in the GWAS.

\begin{tabular}{lllllllllllll}
\hline \multicolumn{1}{c}{ Discovery $(N=9714)$} & & Validation $(N=2662)$ & Combination \\
\hline SNP & Loci & Nearest gene & Allele & MAF & OR $(95 \% \mathrm{Cl})$ & $P$ value & OR $(95 \% \mathrm{Cl})$ & $P$ value & OR $(95 \% \mathrm{Cl})$ & $P$ value \\
rs662799 & $11 \mathrm{q} 23.3$ & APOA5 & A/G & 0.28 & $1.80(1.68-1.94)$ & $5.90 \times 10^{-58}$ & $1.84(1.60-2.11)$ & $7.23 \times 10^{-18}$ & $1.79(1.68-1.91)$ & $1.22 \times 10^{-76}$ \\
rs780092 & $2 \mathrm{p} 23.3$ & GCKR & A/G & 0.36 & $0.77(0.72-0.83)$ & $6.60 \times 10^{-14}$ & $0.70(0.61-0.80)$ & $1.51 \times 10^{-7}$ & $0.77(0.73-0.82)$ & $7.06 \times 10^{-18}$ \\
rs2954031 & $8 q 24.13$ & TRIB1 & T/G & 0.47 & $1.26(1.18-1.34)$ & $1.52 \times 10^{-12}$ & $1.21(1.07-1.37)$ & $2.62 \times 10^{-3}$ & $1.23(1.17-1.30)$ & $9.12 \times 10^{-14}$ \\
rs2075650 & $19 q 13.32$ & TOMM40 & A/G & 0.082 & $1.46(1.31-1.64)$ & $3.25 \times 10^{-11}$ & $1.49(1.20-1.85)$ & $3.29 \times 10^{-4}$ & $1.43(1.30-1.58)$ & $2.53 \times 10^{-13}$ \\
rs1501908 & $5 q 33.3$ & TIMD4 & C/G & 0.27 & $0.81(0.76-0.88)$ & $4.80 \times 10^{-8}$ & $0.80(0.69-0.92)$ & $1.93 \times 10^{-3}$ & $0.84(0.79-0.89)$ & $2.27 \times 10^{-8}$ \\
Imputed & & & & & & & & & & & \\
rs651821 & $11 q 23.3$ & APOA5 & T/C & 0.28 & $1.80(1.68-1.94)$ & $4.75 \times 10^{-57}$ & $1.84(1.60-2.11)$ & $9.46 \times 10^{-18}$ & $1.79(1.68-1.91)$ & $4.52 \times 10^{-76}$ \\
\hline
\end{tabular}

$S N P$ single-nucleotide polymorphism, MAF minor allele frequency, $O R$ odds ratio, $\mathrm{Cl}$ confidence interval.

Manhattan and Q-Q plots). Notably, all SNPs in each of these five loci were clustered, suggesting they do not represent random events. After confirming the independence of these SNPs, we chose the top SNP in each of the five loci: rs780092 (2p23.3), rs1501908 (5q33.3), rs2954031 (8q24.13), rs662799 (11q23.3), and rs2075650 (19q13.32). The validation/replication stage of the GWAS, which was based on an independent set of study participants, confirmed the importance of each SNP, and all SNPs remained significantly associated with hyperlipidemia $(P<0.001$, Table 1, and Supplementary Table 1). The results combined from the two stages confirmed that all five SNPs identified by the GWAS consistently reached statistical significance $\left(5 \times 10^{-8}\right.$, Table 1$)$ We checked these five SNPs in the GWAS catalog database (https:// www.ebi.ac.uk/gwas/) and confirmed that these five known loci are bona fide important in determining hyperlipidemia not only in the Taiwanese population but also in other EAS (Supplementary Table 2).

\section{Variant rs651821 is the most likely functional variant at locus $11 q 23.3$}

Admittedly, the five loci identified by the GWAS are not novel, and rs662799 in 11q23.3 has been identified as the top locus for hyperlipidemia in several studies, including the present one. Variant rs662799 is located upstream of the APOA5 promoter, and
APOA5 is a well-established regulator of blood lipid metabolism ${ }^{19}$. Therefore, we reasoned that APOA5 expression may be regulated by different alleles of rs662799. In other words, lower APOA5 expression and higher TG level is associated with the $\mathrm{G}$ allele of rs662799 (rs662799-G), whereas higher APOA5 expression and lower TG level is associated with the rs662799-A. Not surprisingly, however our analysis as well as previous studies excluded the involvement of rs662799 in APOA5 expression (Supplementary Fig. $1)^{20,21}$. This implied that the downstream gene expression between the fragments containing the G or A allele of rs662799 is not regulated by transcription factors that are predicted to bind this SNP. Therefore, putative causal SNPs at locus 11q23.3 that affect APOA5 level remained to be identified. Toward this end, we imputed all SNPs within a 2-Mb region centered on rs662799 and conducted the analysis again. Variant rs662799 remained the most significant SNP associated with hyperlipidemia, but another SNP, i.e., rs651821, with a high degree of linkage disequilibrium (LD) with rs662799 ( $r^{2}=0.99$, our cohort), attracted our attention because it was the second most significant variant (Fig. $2 a$ and Table 1). Similar to rs662799, rs651821 has been previously associated with TG level ${ }^{8}$. In addition to APOA5, three other apolipoprotein genes, namely $A P O A 1, A P O C 3$ and $A P O A 4$, are located at locus 11q23.3, but the rs651821-C and rs662799-G were only specifically correlated with lower APOA5 expression (Fig. 2b and Supplementary Table 3). 
a
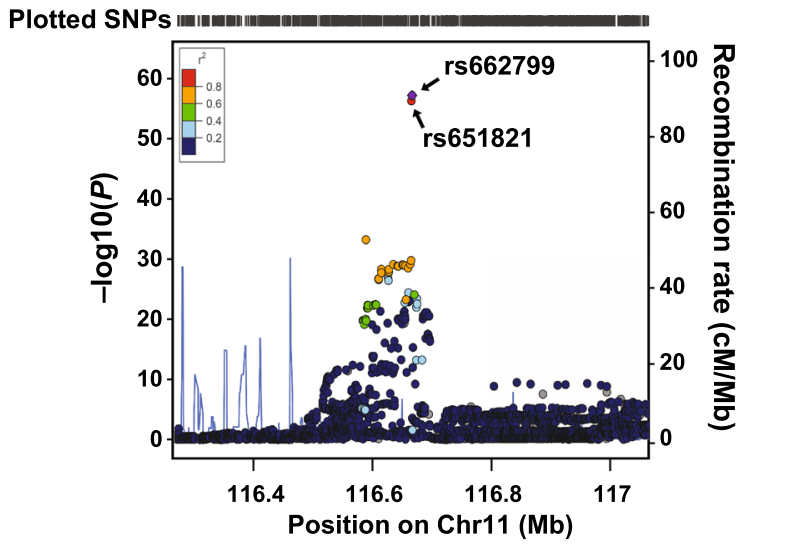

b

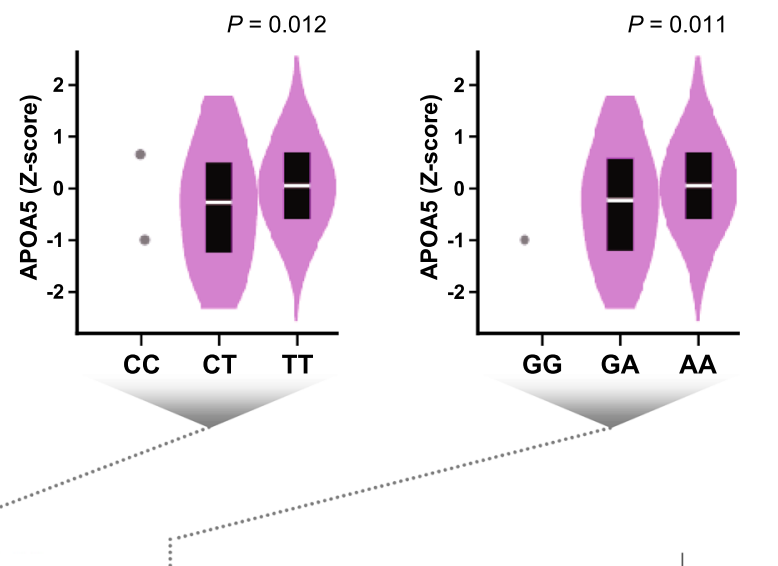

C

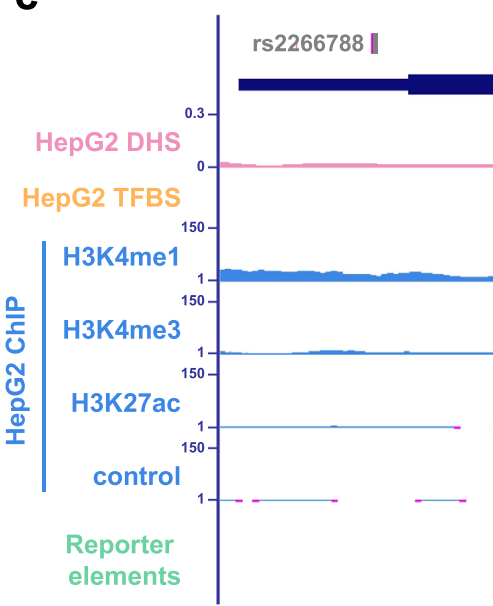

Chr11:116,660,000 
a
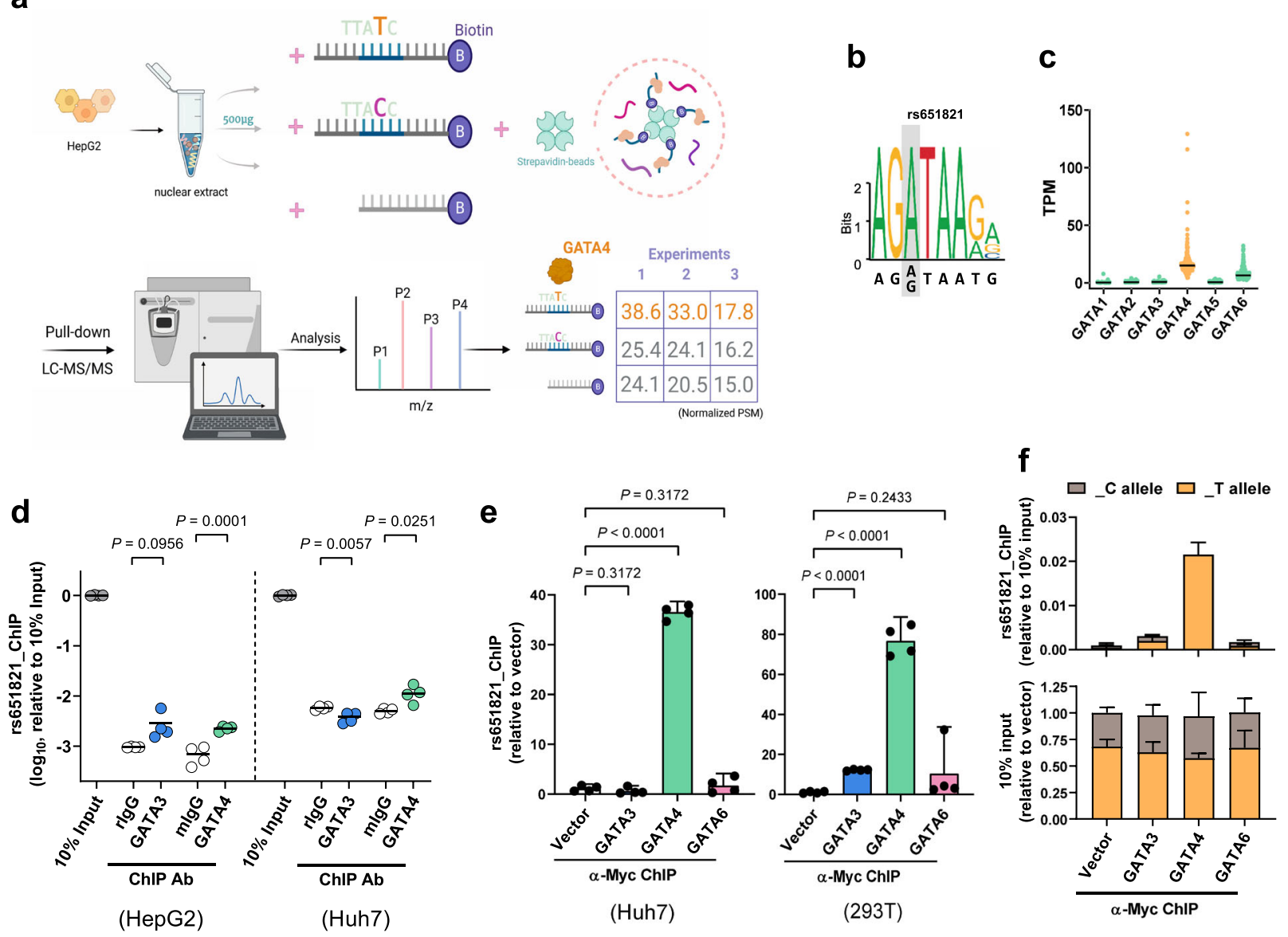

Fig. 3 Identification of transcription factors that preferentially bind rs651821. a Schematic diagram of the mass spectrometry screen for transcription factors that bind rs651821. Enriched peptides corresponding to GATA4 were normalized as indicated. PSM, peptide spectrum matches. The figure was created with BioRender.com. b GATA consensus motif retrieved from the JASPAR 2020 database (http://jaspar. genereg.net/). c Distribution and differential expression of individual members of the GATA family in GTEx liver tissues. d HepG2- and Huh7cell DNA fragments after ChIP with normal rabbit IgG, normal mouse IgG, anti-GATA3, or anti-GATA4 were quantified by SYBR-based qPCR. e Histogram showing the relative rs651821 site enrichment after ChIP with anti-Myc using Huh7 or 293 T cells transfected with pXJ vectors expressing Myc-tagged GATA proteins. f DNA fragments before (lower panel) and after (upper panel) ChIP with anti-Myc performed in 293 T cells (e) were quantified by TaqMan-based qPCR for rs651821. The enriched specific allele in each group was calculated and normalized by the respective FAM ${ }^{\Delta \mathrm{Ct}}$ or $\mathrm{VIC}{ }^{\Delta \mathrm{Ct}}$ of $10 \%$ input, and the result is displayed by the addition of $2^{-\Delta \mathrm{Ct}}$ (FAM $\left.+\mathrm{VIC}\right)$. $P$ values and $95 \% \mathrm{Cl}$ (error bars) for the results within the figure are indicated; three independent experiments of $n=4$ were performed.

quantification (Fig. 3f). Notably, rs651821 is located within APOA5, being at the front of exon 2, but APOA5 expression still could be regulated by the region containing rs651821, as evidenced by results from experiments with CRISPR activation or interference systems with two different proximal single guide RNAs (sgRNAs) targeting the region adjacent to rs651821 (Supplementary Fig. 6). These findings yielded supportive clues that GATA4 can regulate APOA5 expression in liver cells by specifically binding the rs651821-T.

\section{Differential APOA5 regulation by GATA4 depends on the rs651821 genotype}

We next examined whether rs651821 regulates APOA5 expression. Liver cell lines were co-transfected with vector pGL4.23 encoding the T or C allele of rs651821 and a vector expressing GATA4, after which luciferase activity was measured. In HepG2 cells, while the DNA fragments containing rs651821 showed reporter activity compared to empty control, no difference was detected between the two $C$ and T alleles in the absence of GATA4 transfection (Fig. 4a). GATA4 overexpression increased the reporter activity of cells with the rs651821-T (Fig. 4a), and this was also the case for the liver cell lines Huh7 and Huh6 (Fig. 4a and Supplementary Fig. 7a).
In contrast, GATA4 knockdown via a siRNA in these three liver cell lines decreased the reporter activity of the fragment containing the rs651821-T without affecting that of the rs651821-C (Fig. 4b and Supplementary Fig. 7b). Additional experiments with these cell lines carrying the rs651821-TT genotype revealed that GATA4 knockdown significantly downregulated APOA5 expression (Fig. 4c). In contrast, GATA4 knockdown in HA22T/VGH liver cells (carrying the rs651821-CC) did not significantly reduce APOA5 mRNA level (Fig. 4c). These results were consistent with those of our mass spectrometry and ChIP analyses, indicating that GATA4 regulates APOA5 expression in a rs651821 $\mathrm{T}$ allele-specific manner.

Having demonstrated that GATA4 modulates APOA5 expression, we next assessed their genetic interaction in population dataset. The result from the GTEx dataset (Fig. 2b) had already demonstrated significantly greater APOA5 expression for the rs651821-T group than the rs651821-TC group. In fact, upon higher GATA4 expression $(n=95), A P O A 5$ expression was higher for the rs651821-TT group (83 of 95) than the rs651821-TC group (12 of 95). However, when GATA4 was lower expressed $(n=65)$, APOA5 expression did not differ between those two groups (Fig. 4d), implying the involvement of GATA4 in modulating the APOA5 
a

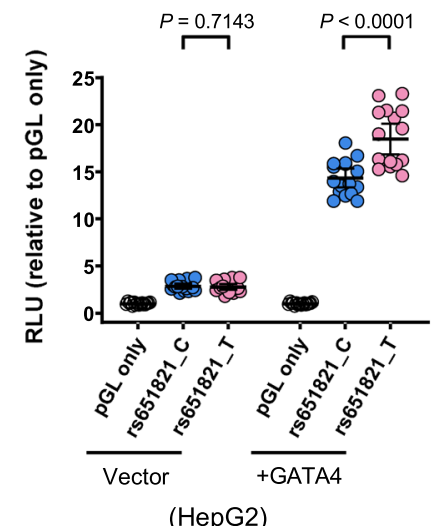

b

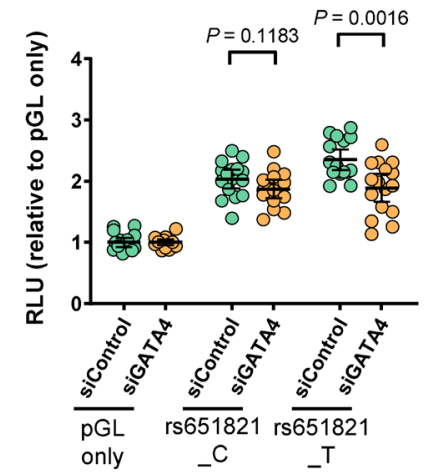

(HepG2)
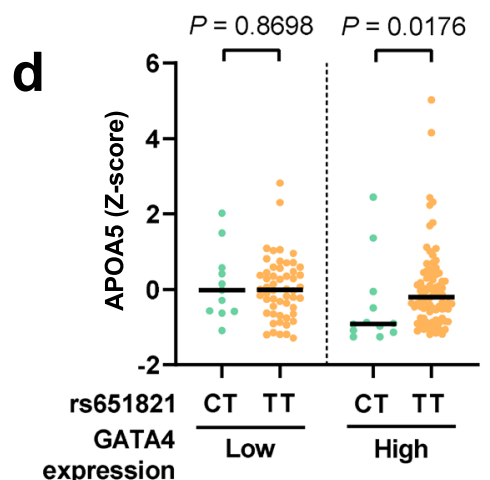
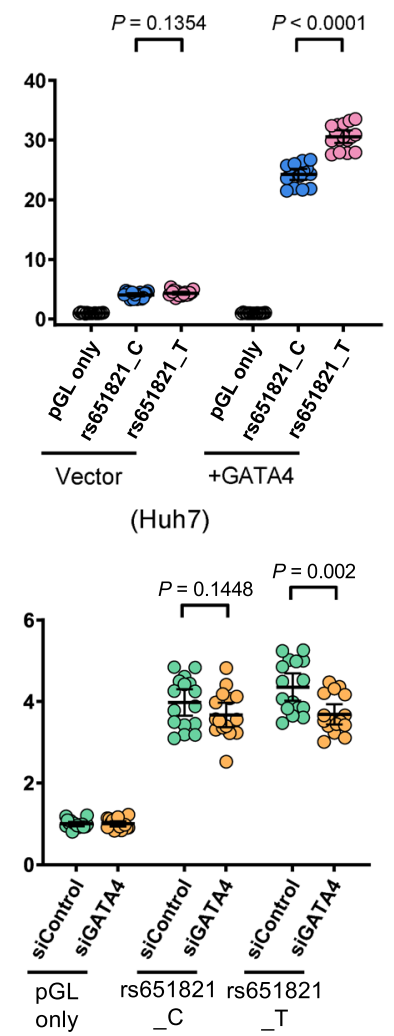

(Huh7)

e

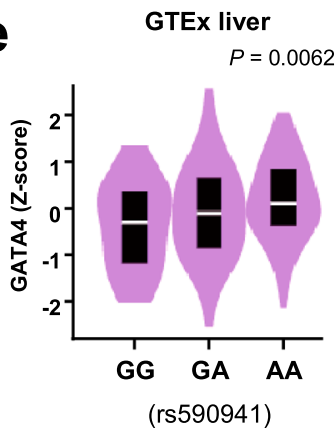

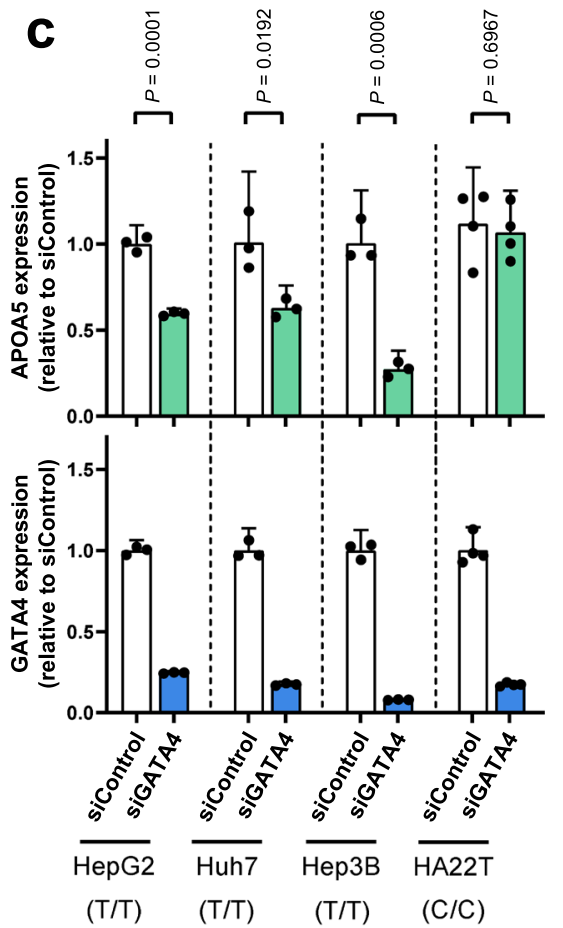

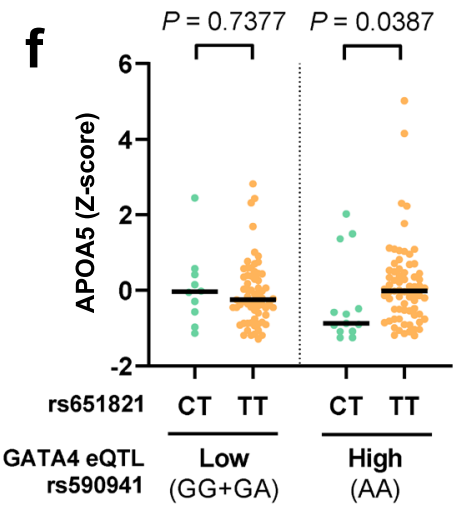

Fig. 4 Allelic imbalance in APOA5 expression is mediated by GATA4. a Reporter analysis of DNA fragments containing different rs651821 genotypes (element B) in HepG2 cells (left) or Huh7 cells (right) transfected with empty vector or GATA4-overexpressing vector. RLU relative luminescence units. b Reporter analysis of DNA fragments containing different rs651821 genotypes in HepG2 cells (left) or Huh7 cells (right) that had been subjected to knockdown by control siRNA or siRNA targeting GATA4. P values and 95\% Cls for the results are indicated; pools of four independent luciferase experiments of $n=4$ are shown as dot plots. c Histogram showing relative GATA4 and APOA5 expression in liver cells transfected with control siRNA or siRNA targeting GATA4, as determined by qPCR. The rs651821 genotype for each cell line was examined by Sanger sequencing as indicated below the graphs. At least two independent transfections of $n=3$ ( $n=4$ in HA22T cells) were performed, and the $P$ values and $95 \% \mathrm{Cls}$ are indicated. d $Z$ scores for APOA5 expression sequentially stratified by GATA4 expression and rs651821 genotypes based on the information retrieved from the GTEx liver dataset. Median values are indicated. Statistical difference between two groups was determined by the Mann-Whitney $U$ test. e The significant GATA4 eQTL in liver tissues queried from the GTEx dataset. $\mathbf{f} Z$ scores for APOA5 expression sequentially stratified by rs590941 and rs651821 genotypes and analyzed as described in (d).

eQTL. To further confirm our hypothesis, we searched for a SNP that represented GATA4 expression in liver tissue, according to GTEx (Supplementary Dataset 1). This revealed that rs590941 is conditionally the most significant cis-eQTL for GATA4 (Fig. 4e). The rs590941-AA genotype is associated with higher cellular level of GATA4 in liver, implying GATA4 activation. The observed greater APOA5 expression in the rs651821-TT group $(n=64)$ than in the rs651821-TC group $(n=10)$ remained statistically significant in the samples carrying the rs590941-AA genotype, indicating a genetic contribution from GATA4 expression (Fig. 4f). However, for the
rs590941-GG/GA genotype $(n=82)$, which is associated with lower GATA4 expression, APOA5 expression was not significantly higher in the rs651821-TT group $(n=69)$ (Fig. 4f). Notably, APOA5 expression did not correlate with rs590941 (the cis-eQTL for GATA4) (Supplementary Fig. 8). Therefore, the discriminate role of rs590941 in modulating the association between rs651821 and APOA5 expression probably reflects direct regulation by GATA4. In total, the findings suggested a role for rs651821 in modulating $A P O A 5$ expression via allele-specific recruitment of GATA4 to the APOA5 promoter. 

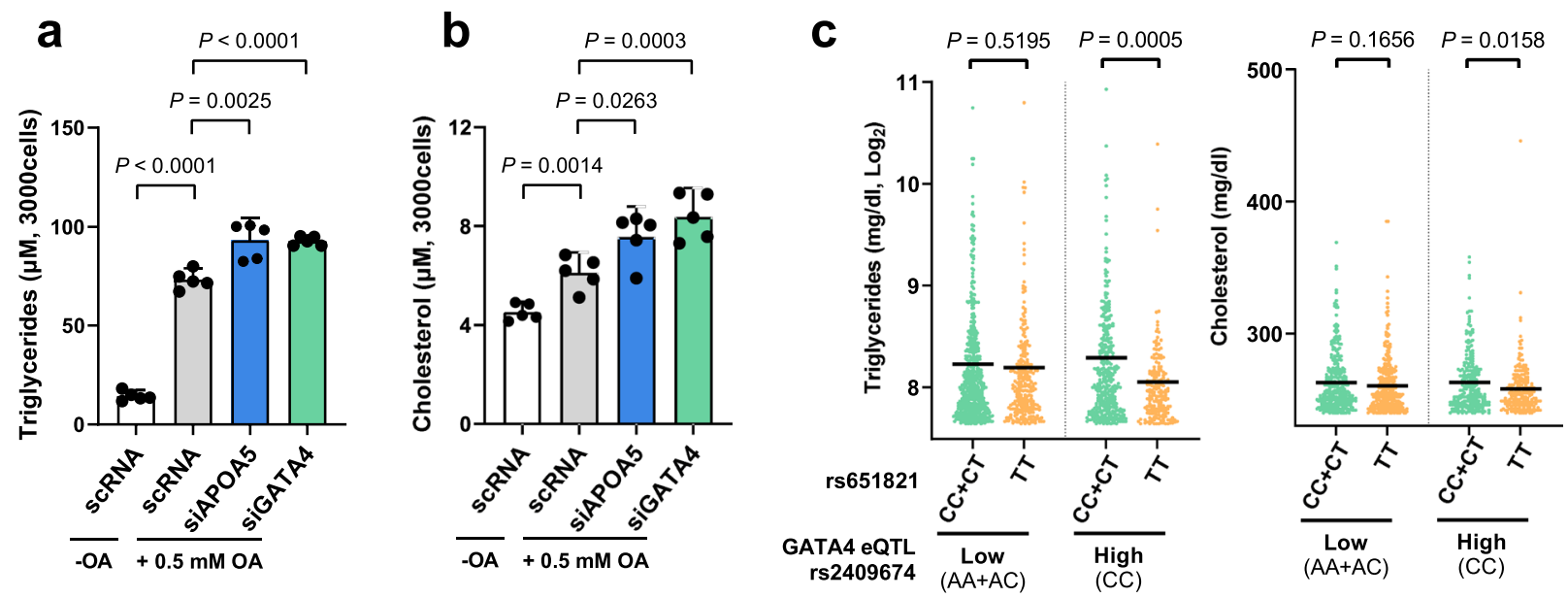

d
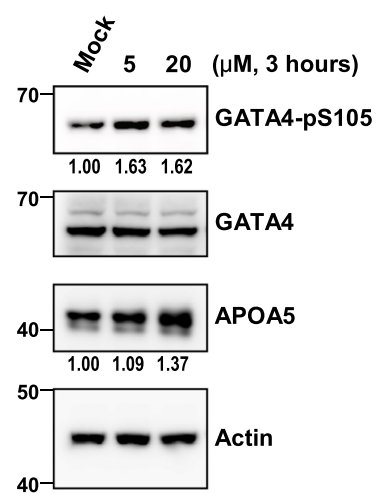

(HepG2)

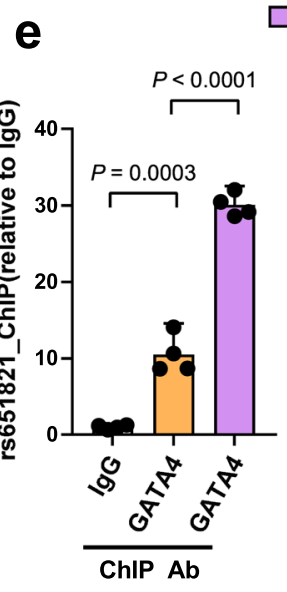

(HepG2) +phenylephrine

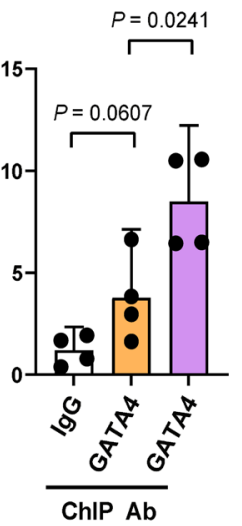

(Huh7)

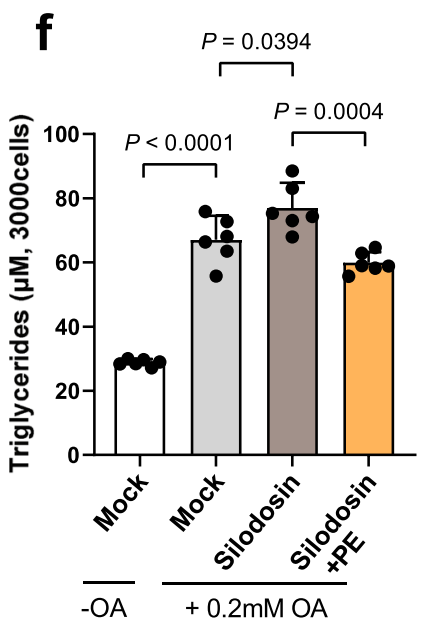

Fig. 5 Regulatory role of GATA4 in cellular and plasma TG homeostasis. a, b Quantification of TG (a) or TC (b) level in HepG2 cells treated with or without oleic acid (OA). $P$ values and $95 \%$ Cls are indicated, and independent measurements of $n=5$ are plotted. c Data for participants with plasma TG $\geq 200 \mathrm{mg} / \mathrm{dl}$ (left) and plasma TC $\geq 240 \mathrm{mg} / \mathrm{dl}$ (right) were retrieved from the TWB cohort and sequentially stratified by the rs 2409674 and rs651821 genotypes. Mean values are indicated. Statistical difference between two groups was determined by the Student's $t$ test. d Immunoblot showing APOA5 expression and phosphorylation of GATA4 at serine 105 following treatment of HepG2 cells to phenylephrine. All blots derive from the same experiment and were processed in parallel. e Histogram showing the relative rs651821 site enrichment after ChIP with normal mouse IgG or anti-GATA4 using HepG 2 and Huh7 cells treated with or without $20 \mu \mathrm{M}$ phenylephrine for $3 \mathrm{~h}$. f Quantification of OA-induced TG level in HepG2 cells co-treated with or without $10 \mathrm{nM}$ silodosin and $20 \mu \mathrm{M}$ phenylephrine. $P$ values and $95 \% \mathrm{Cls}$ are indicated, and independent measurements of $n=6$ are plotted.

\section{Elevated GATA4 contributes to TG homeostasis}

Given that knockout of Apoa5 in mice increases plasma TG level, and mutation at $A P O A 5$ gene causes hypertriglyceridemia and elevated TC ${ }^{19,22}$, GATA4 may affect TG and TC homeostasis through APOA5. We treated HepG2 cells with OA, which causes lipid accumulation and steatosis, and monitored changes in the levels of TG and TC. Knockdown of APOA5 or GATA4 resulted in significant accumulation of both TG (Fig. 5a) and TC (Fig. 5b) in cells. To confirm the clinical relevance between GATA4-induced APOA5 upregulation and TG regulation, we divided our TWB cohort into two groups by GATA4 eQTL. The rs590941-G is carried by relatively few Taiwanese individuals compared with EUR $(5.3 \%$ in the TWB vs. $29 \%$ in EUR). Therefore, for subsequent analysis, we chose another GATA4 eQTL, namely rs2409674 (Supplementary Fig. 9), with a higher proportion of the minor allele (39.6\%) in our population. The results showed that rs651821-TT imparted no significant benefit on plasma TG or TC level for the TWB participants carrying rs2409674-AA/AC, the low-expressing GATA4 eQTL (Fig. 5c). However, the combination of rs651821-TT and elevated GATA4 expression (rs2409674-CC) conferred a benefit on plasma TG and TC level (Fig. 5C). These data further confirmed the causal role of rs651821 in the association with hyperlipidemia by mediating allele-specific involvement of GATA4 at APOA5 expression.

In cardiac myocytes, GATA4 activity is induced by phenylephrine through phosphorylation at serine $105^{23}$. Phenylephrine is a nonselective agonist for $a_{1}$-adrenergic receptors ${ }^{24}$. As the $a_{1 A^{-}}$ adrenergic receptor (encoded by $A D R A 1 A$ ) is highly expressed and more abundant than the other a1 subtypes in liver, (Supplementary Fig. 10), we observed phenylephrine promoted APOA5 expression, as well as GATA4 serine 105 phosphorylation, in HepG2 cells (Fig. 5 d). This finding result can be explained by ChIP analysis that phenylephrine treatment enhanced GATA4 enrichment at rs651821 in liver cells (Fig. 5e). Moreover, lipid accumulation caused by $\mathrm{OA}$ and silodosin, the selective inhibitor of $a_{1 A}$-adrenergic receptor, could be alleviated by administration of phenylephrine (Fig. 5f). Collectively, this suggests a therapeutic model for the synergistic effect of APOA5 and GATA4 on TG level, 


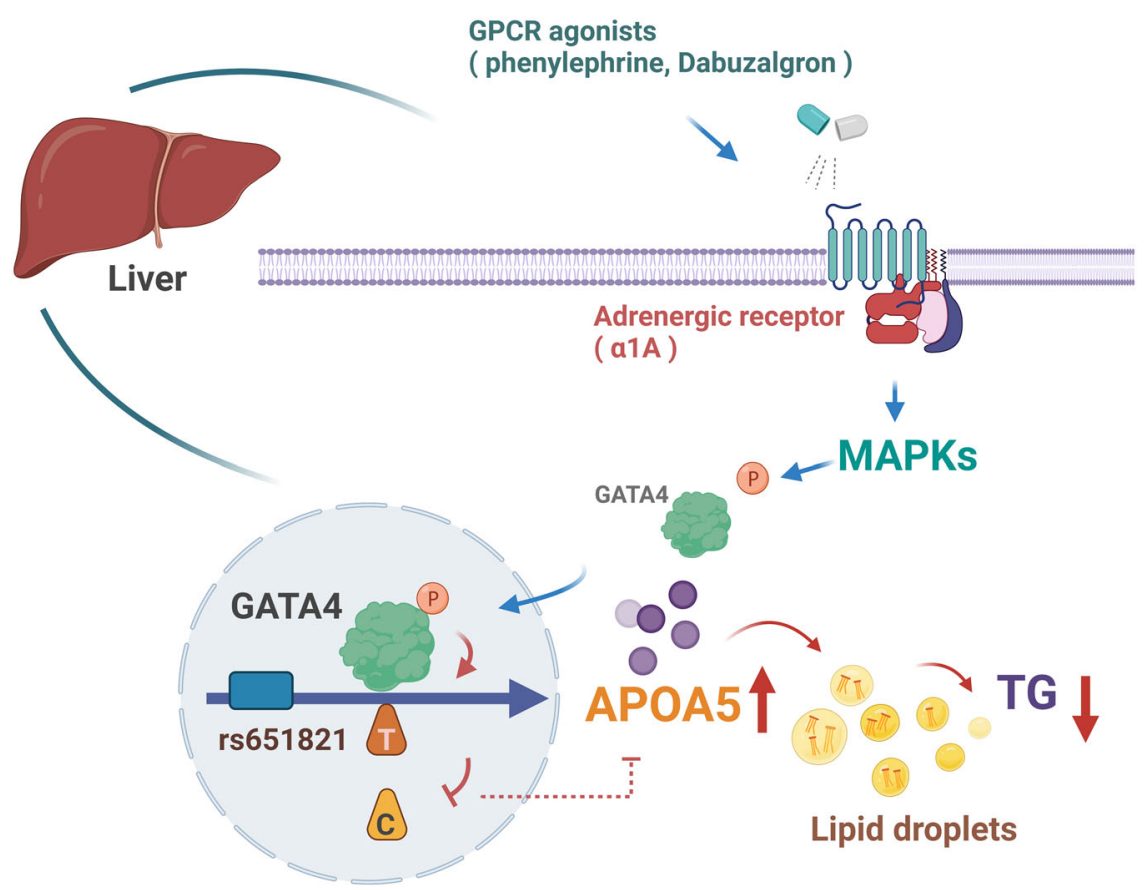

Fig. 6 Model of the regulatory mechanism for rs651821 mediating APOA5 expression through GATA4, thereby promoting TG hydrolysis in liver. The figure was created with BioRender.com.

which may help control an individual's susceptibility to hyperlipidemia and other metabolic abnormalities (Fig. 6).

\section{DISCUSSION}

Given that abnormal levels of TG and TC constitute a threat to human health worldwide, our findings have both etiological importance and translational relevance. Explicitly, we demonstrate a causal role for GATA4 in modulating APOA5 level. Our study suggests standard avenues for demonstrating how to utilize GWAS results to identify cellular pathways that can be modulated via pharmacological means.

Among these hyperlipidemia-associated five loci, a mechanism has been established for only rs780092 (2p23.3), owing to LD with rs780094 ( $r^{2}=0.45$ in EAS of the 1000 Genomes Project); rs780094 facilitates the binding of transcription factor FOXA2 to regulate GCKR expression ${ }^{16}$. The rs2954031 (8q24.13) has been explained by its LD-SNP, rs2001844, which was reported as an eQTL for TRIB1 gene ${ }^{25}$, although how TRIB1 regulates TG remains unclear. The rs2075650 (19q13.32) is located in an intron of TOMM40, and rs1501908 (5q33.3) is located between TIMD4 and HAVCR1. However, these two SNPs do not act as the cis-eQTL for adjacent genes in GTEx liver tissue. The locus 11q23.3, led by rs662799, is the most significant locus consistently identified. Given that the molecular mechanism by which this locus contributes to metabolic disease remains unclear, our current study as well as several others have attempted to determine the functional significance of rs66279920,21, but this SNP has not been definitively linked to the transcription-promoting activity of APOA5. Of special note here is that interferon regulatory factors (IRFs) were predicted to repress APOA5 through the rs662799-G, but we failed to observe any allele-specific modulation of luciferase activity by IRFs (Supplementary Fig. 1). Apparently, rs662799 is not the causal variant. Instead, another SNP LD with and adjacent to rs662799, namely rs2266788, were then expected. The rs 2266788 was reported its interaction with two miRNAs, namely miR-3201 and miR-485-5 $\mathrm{p}^{26,27}$. These two miRNAs were identified based on their sequence complementary with the rs2266788 region (Supplementary Fig. 11). However, these two miRNAs are actually undetectable in GTEx liver tissue, which argues against their participation in regulating APOA5 level in liver. Additionally, miR3201 reportedly represses APOA5 expression through the rs2266788-A, the allele that is thought to be associated with higher APOA5 level ${ }^{26}$. This obviously conflicts with the current paradigm. For miR-485-5p, neither allele of rs2266788 is targeted with high complementarity by the seed region of miR-485-5p (Supplementary Fig. 11), strongly weakening the probability that the miR-485-5p modulates APOA5 expression. Genetically, rs662799 is in almost complete LD with rs651821 $\left(r^{2}=0.99\right)$ but not rs2266788 $\left(r^{2}=0.75\right)$. Thus, we propose rs651821 is more likely to be the functional variant within locus $11 \mathrm{q} 23.3$.

APOA5 interacts with very low-density lipoprotein (VLDL), the major carrier of TG, and decreases the TG level in circulating VLDL by enhancing lipoprotein lipase activity ${ }^{28}$. As a result, loss-offunction mutations in $A P O A 5$ cause severe hypertriglyceridemia $^{22,28}$. Similarly, mutations in LPL (lipoprotein lipase), APOC2, GPIHBP1, and $L M F 1$, and the recently identified transcription factor CREB3L3, are also found to contribute to the development of severe hypertriglyceridemia ${ }^{6,29}$. Although these rare mutations are important, they account for at most $15.2 \%$ of the etiology of hypertriglyceridemia, yet $>32 \%$ of patients having severe hypertriglyceridemia are estimated to be affected by a combination of common variants ${ }^{6}$. Hence, polygenic risk scores, which have been mainly based on EUR, are currently used to predict the development of complex phenotypes, such as hyperlipidemia and abnormal TG and TC levels ${ }^{6}$. However, it should be acknowledged that certain common variants-with rs651821 being a good example-is more prevalent in the EAS (29\%) than that in other populations in the world (only $8 \%$ in the EUR). That explains why this variant has not been thoroughly investigated in previous GWAS conducted with EUR, and, more critically, why the involvement of GATA4 in regulating APOA5 as well as plasma TC and TG levels has seldom been appreciated.

Our study showed insufficient GATA4 activity alters the regulation of TG level in cells and in clinical samples. This finding suggests that GATA4 agonist(s), such as phenylephrine, could be developed to treat hyperlipidemia, and exploring the transcriptomic change affected by phenylephrine would be crucial to 
understand the possible therapeutic action through GATA4 activity. While phenylephrine has shown therapeutic potential to downregulate hepatic TG level in animal models $\mathrm{s}^{30}$, and, supported by the findings of the present study, phenylephrine may increase blood pressure clinically by promoting vasoconstriction ${ }^{31}$, an unfavorable side effect for hyperlipidemia patients. To overcome this side effect, one $a_{1 A}$-adrenergic agonist, Ro 115-1240 (dabuzalgron), has demonstrated no effect on blood pressure and heart rate in clinical study and would expect to be much better choice to treat hyperlipidemia ${ }^{32,33}$. In conclusion, better understanding of the mechanisms underpinning this process is certainly needed to develop personalized preventive and therapeutic strategies for controlling hyperlipidemia and abnormal TC level.

\section{METHODS \\ Study cohort}

A total of 23,988 samples with genotype and biochemical data were collected from the TWB (Supplementary Table 4). Individuals who had been diagnosed with hyperlipidemia (self-reported) or had a measured TG of $\geq 200 \mathrm{mg} / \mathrm{dl}$ or $\mathrm{TC} \geq 240 \mathrm{mg} / \mathrm{dl}$ were defined as hyperlipidemia cases, and other individuals who matched the criteria of $\mathrm{TG}<150 \mathrm{mg} / \mathrm{dl}$ and $\mathrm{TC}<200 \mathrm{mg} / \mathrm{dl}$ were selected as the control group. Individuals who had intermediate measures were excluded. The discovery stage of the study included 18,419 individuals, among whom 3310 were selected as cases and 6404 as controls. An additional 829 cases and 1833 controls from the remaining non-overlapped 5569 individuals were included in the validation stage. The study was approved by the ethics committee of the institutional review board of Academia Sinica (AS-IRB01-16018) with informed consent that was obtained from each participant in accordance with the institutional requirements and the Declaration of Helsinki principles.

\section{Genotyping and association analysis}

Blood DNA samples from the study participants were genotyped using the Affymetrix TWB SNP chip, as described ${ }^{18}$. For the SNP quality-control process, we selected SNPs with a call rate $\geq 95 \%, P$ value for Hardy-Weinberg equilibrium (HWE) of $\geq 1 \times 10^{-4}$, and minor allele frequency $(\mathrm{MAF}) \geq 5 \%$. In total, 597,758 SNPs were selected for the association analysis. Quality-control for genotypes and biochemical measures was performed with Plink software ${ }^{34}$, and odds ratios for individual SNPs associated with hyperlipidemia were calculated using logistic regression with adjustment for the effect of age, sex, body mass index, and principal components. Significance was defined according to the conventional genome-wide significance threshold $\left(5 \times 10^{-8}\right)$, and SNPs located within a $\pm 500 \mathrm{~kb}$ region of a significant SNP were considered as a part of that locus. SNPs were further imputed with SHAPEIT and IMPUTE2 software using EAS data from Phase 3 (Version 5) of the 1000 Genomes Project as the reference panel, as described ${ }^{35}$. SNPs with a posterior probability of $<0.9$, call rate $<0.01, \mathrm{MAF}<1 \%$, or HWE $P$ value of $<1 \times 10^{-6}$ were excluded, and the other SNPs were plotted to visualize the association using LocusZoom ${ }^{36}$.

\section{Analysis of data from the GTEx portal}

The transcripts per million (TPM) normalized RNA sequences data for genes and the expression quantitative trait loci (eQTL) results in liver samples were retrieved from GTEx Analysis V8 (www.gtexportal.org). The statistical significance was determined using the website's algorithm ${ }^{37}$. The top cis-eQTL for GATA4 was initially selected from 40,414 SNPs that are located within $\pm 1 \mathrm{Mb}$ of the transcription start site of GATA4. Because smaller sample sizes restrict minor allele stratification, we excluded those candidate SNPs with MAF $<10 \%$ (Supplementary Dataset 1). In consequence, rs590941 was the top eQTL with the lowest $P$ value of 0.0062 . To assess any genetic interaction between rs651821 and rs590941, gene expression data and genotypes for rs651821 and rs590941 in livertissue samples from 156 individuals were accessed from GTEx protected data in dbGaP (Database of Genotypes and Phenotypes), and APOA5 and GATA4 expression data were transformed to a $z$ score before analysis.

\section{Cell culture}

Human liver cell lines HepG2 (RRID:CVCL_0027), cultured in Eagle's minimum essential medium (\#M0643, Sigma-Aldrich), and HA22T (synonyms HA22T/VGH, RRID:CVCL_7046), cultured in DME medium (\#D5648, Sigma-Aldrich) supplemented with $0.1 \mathrm{mM}$ non-essential amino acids, were purchased from the Bioresource Collection and Research Center (Hsinchu, Taiwan). Human liver cell lines Hep3B (synonyms Hep3B2.1-7, RRID:CVCL_0326) and Huh7 (RRID:CVCL_0336) were obtained from Dr. HuiChun Wang (Kaohsiung Medical University), and Huh6 (RRID:CVCL_4381) was obtained from Dr. Chia-Hung Yen (Kaohsiung Medical University). These cells were cultured in DME medium. Human embryonic kidney 293 T cells (synonyms HEK293T, RRID:CVCL_0063) were as previously described $^{35}$. All cells were maintained in medium supplemented with $10 \%$ fetal bovine serum (\#26140, Thermo Fisher Scientific). All cell lines were confirmed to be mycoplasma-free and authenticated within the last three years using the short-tandem repeats profiling method (Promega GenePrint 24 System) by Genelabs (Taipei, Taiwan).

\section{Treatment and transfection}

Oleic acid (OA) sodium salt (\#O7501) and phenylephrine (\#P6126) were purchased from Sigma-Aldrich, and silodosin (\#6663) was obtained from Tocris Bioscience. For the in vitro model of hepatic steatosis, OA was dissolved in $99 \%$ methanol as a stock solution ${ }^{38}$. Before treatment, HepG2 cells were washed twice with Hank's balanced salt solution (\#H1387, Sigma-Aldrich) and then incubated in phenol red-free DME medium (\#D2902, Sigma-Aldrich) containing 1\% bovine serum albumin and $10 \%$ charcoal-stripped fetal bovine serum (\#12676, Thermo Fisher Scientific).

Cells were transfected with plasmids using TransIT-X2 (Mirus), whereas transfection with small interfering RNAs (siRNAs) utilized Lipofectamine RNAiMAX (Thermo Fisher Scientific). Full-length CDNAs encoding GATA3, GATA4, GATA6, FOXA1, TCF7L2, IRF1, IRF2 and IRF3 were amplified from human cell-line cDNAs and cloned into the Myc-tagged pXJ vector, and expression in mammalian cells was assessed via immunoblotting with antiMyc (1:3000, \#M4439, Sigma-Aldrich). The other antibodies used for immunoblotting were: anti-GATA4 (1:500, \#MA5-15532), anti-phosphoGATA4 (1:500, \#44-948), and anti-APOA5 (1:1500, \#MA1-16809) from Thermo Fisher Scientific; anti-actin (1:2000, \#A2066), and anti-a-tubulin (1:10000, \#T6199) from Sigma-Aldrich. Supplementary Table 5 lists the primer pairs used for plasmid construction. The control siRNA has been described ${ }^{39}$. GATA4 siRNA pools (\#s5603, \#s535120 and \#s535121) and APOA5 siRNA (\#s528181) were obtained from Thermo Fisher Scientific.

\section{Luciferase reporter assay}

All reporter fragments were amplified using 293T genomic DNA to obtain two distinct genotypes and then cloned into vector pGL4.23 (\#E8411, Promega). Supplementary Table 5 lists the primer pairs used for reporterfragment construction. For reporter analysis, pGL4.23 constructs contained fragments with the major or minor allele of each of rs662799 or rs651821 of the pGL4.23 vector was left empty (control). HepG2 (40,000 cells/well), Huh7 (35,000 cells/well), or Huh6 (15,000 cells/well) in 24-well plates were co-transfected with expression vectors or siRNAs. pRL-tk, which encodes Renilla luciferase, was co-transfected as an internal control. After $48 \mathrm{~h}$ of incubation, reporter activities were determined using the Dual-Luciferase Reporter Assay System (\#E1960, Promega) and normalized to the background activity measured in cells transfected with empty pGL4.23 35,39 . The mean and $95 \%$ confidence interval (Cl) for each group was calculated based on the pools of four independent experiments, each with four replicates from independent wells. The statistical significance of differences was determined using the Student's $t$ test.

\section{Mass spectrometry}

The 5'-biotinylated oligonucleotide duplexes are indicated in Supplementary Table 5. Briefly, nuclear extract from HepG2 cells was prepared using the NE-PER Nuclear and Cytoplasmic Extraction kit (Thermo Fisher Scientific). The reaction was incubated at room temperature in $500 \mu \mathrm{l}$ binding buffer containing $10 \mathrm{mM}$ Tris- $\mathrm{HCl}(\mathrm{pH} 7.5), 50 \mathrm{mM} \mathrm{KCl}, 10 \mathrm{mM}$ $\mathrm{MgCl}_{2}, 10 \%$ glycerol, and $1 \mathrm{mM}$ DTT. In each reaction, $500 \mathrm{pmol}$ of a different probe duplex was incubated with $500 \mu \mathrm{g}$ nuclear extract plus $5 \mu \mathrm{g}$ poly(dl:dC) (\#P4929, Sigma-Aldrich) and $5 \mu \mathrm{g}$ poly(dA:dT) (\#P9764, Sigma-Aldrich). During a $90-\mathrm{min}$ incubation, $30 \mu \mathrm{l}$ of streptavidin Sepharose beads (\#17511301, Cytiva) was added with additional incubation for $30 \mathrm{~min}$. The pulldown matrix coupled to beads was then washed 
four times with $500 \mu \mathrm{l}$ wash buffer $(20 \mathrm{mM}$ Tris- $\mathrm{HCl} \mathrm{pH} 7.5,500 \mathrm{mM} \mathrm{NaCl}$, $0.2 \mathrm{mM}$ EDTA). Proteins were eluted and analyzed by liquid chromatography coupled with tandem mass spectrometry (carried out by Biotools Company, New Taipei City, Taiwan). The experiments were performed three times using independently prepared batches of HepG2 nuclear extract, and label-free proteins from the identified peptides were quantified based on normalized peptide-spectra matches (Supplementary Dataset 2).

\section{Quantitative PCR (qPCR) for gene expression and chromatin immunoprecipitation (ChIP)}

Gene expression of APOA5 or GATA4 was quantified via reversetranscription $\mathrm{qPCR}^{35,39}$. To determine whether the $\mathrm{rs} 651821$ region could serve as an enhancer, mRNA was extracted from HepG2 cells that had been co-transfected using TransIT-X2 with pcDNA3.3-dCas9-VPR or p5w-dCas9KRAB and a pU6-sgRNA vector encoding sgEGFP, sgRNA1, or sgRNA2 sequence ${ }^{35}$. Supplementary Table 5 lists the primer pairs used for CDNA quantification and sgRNA construction. Relative gene expression was normalized to that for TBP using the comparative CT method. Three independent experiments were performed, each with three experimental replicates, and mean and $95 \% \mathrm{Cl}$ values were calculated using the Student's $t$ test.

ChIP was performed using the EZ-Magna ChIP G kit (\#17-611, MerckMillipore) according to previous studies ${ }^{35,39}$. HepG2 $\left(6 \times 10^{6}\right.$ cells/dish) or Huh7 $\left(3 \times 10^{6}\right.$ cells/dish) cells in $100 \mathrm{~mm}$ dishes were fixed in $1 \%$ formaldehyde, and DNA was sonicated with Bioruptor Pico (Diagenode). Sheared DNA was then immunoprecipitated with $1 \mu \mathrm{g}$ ChIP-grade antibody: anti-GATA3 (\#ab199428, Abcam) or anti-GATA4 (\#MA5-15532). Normal rabbit or mouse IgG served as the control antibody. Immunoprecipitated DNA was eluted and then quantified via SYBR qPCR (\#A25741, Thermo Fisher Scientific). For those cells overexpressing Myc-tagged GATA family members, sheared DNA was immunoprecipitated with anti-Myc (\#M4439). Allele-specific quantification of eluted DNA in $293 \mathrm{~T}$ cells was determined with TaqMan ${ }^{\mathrm{TM}}$ Fast Advanced Master Mix (\#4444557, Thermo Fisher Scientific) with the specific fluorescence probe set described in Supplementary Table 5.

\section{Measurement of intracellular TG and TC}

TG and TC content in steatotic HepG2 cells was determined with the Triglyceride-Glo Assay (\#J3160, Promega) and Cholesterol/Cholesterol Ester-Glo Assay, (\#J3190, Promega), respectively. Briefly, HepG2 cells that had been subjected to siRNA-mediated mRNA knockdown were seeded in a 96-well plate ( 3000 cells/well) and treated with $0.5 \mathrm{mM}$ OA for $24 \mathrm{~h}$. The medium was removed, and cells were washed twice by Hank's balanced salt solution and lysed with $30 \mu \mathrm{l}$ buffer included in the kits. The concentration of each of TG and TC was calculated based on standard curves. Mean and $95 \% \mathrm{Cl}$ values for each experimental group were calculated from five independent wells, and three independent experiments were performed. The statistical significance was determined using the Student's $t$ test.

\section{Reporting summary}

Further information on research design is available in the Nature Research Reporting Summary linked to this article.

\section{DATA AVAILABILITY}

The expression vectors generated during the current study are available from the corresponding author on request. The GWAS summary statistics are deposited in the GWAS catalogue https://www.ebi.ac.uk/gwas/ (accession numbers: GCST90090994) and are openly available in figshare at https://doi.org/10.6084/m9.figshare.14897097. Individual data are used under license for the current study, and so are not publicly available. Data are, however, available directly from the Taiwan Biobank (biobank@gate.sinica.edu.tw) pending permission from the Ministry of Health and Welfare, Taiwan. Mass spectrometry was carried out by Biotools Company and the proteomics RAW files are unavailable. However the quantification of label-free proteins based on normalized peptide-spectra matches are available within the article and its Supplementary Dataset 2. Public datasets that support the findings of this study are available from GTEx portal, https://www.gtexportal.org/home/datasets; Genomics of Drug Sensitivity in Cancer Project, https://www.cancerrxgene.org/downloads/ bulk_download; Cancer Cell Line Encyclopedia, https://portals.broadinstitute.org/ ccle/data; and in ArrayExpress, https://www.ebi.ac.uk/arrayexpress/experiments/EMTAB-2706/.

Received: 2 July 2021; Accepted: 16 December 2021; Published online: 19 January 2022

\section{REFERENCES}

1. Hsiung, C. N. et al. The causal relationship of circulating triglyceride and glycated hemoglobin: a mendelian randomization study. J. Clin. Endocrinol. Metab. 105, 908-919 (2020).

2. Johansen, C. T., Kathiresan, S. \& Hegele, R. A. Genetic determinants of plasma triglycerides. J. Lipid Res. 52, 189-206 (2011).

3. Triglyceride Coronary Disease Genetics Consortium and Emerging Risk Factors Collaboration. Triglyceride-mediated pathways and coronary disease: collaborative analysis of 101 studies. Lancet 375, 1634-1639 (2010).

4. Wannamethee, G. \& Shaper, A. G. The association between heart rate and blood pressure, blood lipids and other cardiovascular risk factors. J. Cardiovasc Risk 1 223-230 (1994).

5. National Cholesterol Education Program Expert Panel on Detection, E. \& Treatment of High Blood Cholesterol in, A. Third Report of the National Cholesterol Education Program (NCEP) expert panel on detection, evaluation, and treatment of high blood cholesterol in adults (Adult Treatment Panel III) final report. Circulation, 106, 3143-3421 (2002).

6. Dron, J. S. et al. Severe hypertriglyceridemia is primarily polygenic. J. Clin. Lipido. 13, 80-88 (2019)

7. Teslovich, T. M. et al. Biological, clinical and population relevance of 95 loci for blood lipids. Nature 466, 707-713 (2010).

8. Willer, C. J. et al. Discovery and refinement of loci associated with lipid levels. Nat. Genet. 45, 1274-1283 (2013).

9. Spracklen, C. N. et al. Association analyses of East Asian individuals and transancestry analyses with European individuals reveal new loci associated with cholesterol and triglyceride levels. Hum. Mol. Genet. 26, 1770-1784 (2017).

10. Hindorff, L. A. et al. Potential etiologic and functional implications of genomewide association loci for human diseases and traits. Proc. Natl Acad. Sci. USA 106, 9362-9367 (2009).

11. Freedman, M. L. et al. Principles for the post-GWAS functional characterization of cancer risk loci. Nat. Genet. 43, 513-518 (2011).

12. Choi, J. et al. A common intronic variant of PARP1 confers melanoma risk and mediates melanocyte growth via regulation of MITF. Nat. Genet. 49, 1326-1335 (2017).

13. Beesley, J. et al. eQTL colocalization analyses identify NTN4 as a candidate breast cancer risk gene. Am. J. Hum. Genet. 107, 778-787 (2020).

14. Pan, G. et al. rs953413 regulates polyunsaturated fatty acid metabolism by modulating ELOVL2 expression. iScience 23, 100808 (2020).

15. Davis, J. P. et al. Enhancer deletion and allelic effects define a regulatory molecular mechanism at the VLDLR cholesterol GWAS locus. Hum. Mol. Genet. 28 888-895 (2019).

16. Lopez Rodriguez, M. et al. Identification and characterization of a FOXA2 regulated transcriptional enhancer at a type 2 diabetes intronic locus that controls GCKR expression in liver cells. Genome Med. 9, 63 (2017).

17. Musunuru, K. et al. From noncoding variant to phenotype via SORT1 at the $1 \mathrm{p} 13$ cholesterol locus. Nature 466, 714-719 (2010).

18. Chen, C. H. et al. Population structure of Han Chinese in the modern Taiwanese population based on 10,000 participants in the Taiwan Biobank project. Hum Mol. Genet. 25, 5321-5331 (2016).

19. Pennacchio, L. A. et al. An apolipoprotein influencing triglycerides in humans and mice revealed by comparative sequencing. Science 294, 169-173 (2001).

20. Palmen, J. et al. The functional interaction on in vitro gene expression of APOA5 SNPs, defining haplotype APOA52, and their paradoxical association with plasma triglyceride but not plasma apoAV levels. Biochim. Biophys. Acta 1782, 447-452 (2008).

21. Talmud, P. J., Palmen, J., Putt, W., Lins, L. \& Humphries, S. E. Determination of the functionality of common APOA5 polymorphisms. J. Biol. Chem. 280, 28215-28220 (2005).

22. Mendoza-Barbera, E. et al. Structural and functional analysis of APOA5 mutations identified in patients with severe hypertriglyceridemia. J. Lipid Res. 54, 649-661 (2013).

23. Morimoto, T. et al. Phosphorylation of GATA-4 is involved in a1-adrenergic agonist-responsive transcription of the endothelin-1 gene in cardiac myocytes. J. Biol. Chem. 275, 13721-13726 (2000).

24. Aries, A., Paradis, P., Lefebvre, C., Schwartz, R. J. \& Nemer, M. Essential role of GATA-4 in cell survival and drug-induced cardiotoxicity. Proc. Natl Acad. Sci. USA 101, 6975-6980 (2004). 
25. Douvris, A. et al. Functional analysis of the TRIB1 associated locus linked to plasma triglycerides and coronary artery disease. J. Am. Heart Assoc. 3, e000884 (2014).

26. Cui, G. et al. A functional variant in $A P O A 5 / A 4 / C 3 / A 1$ gene cluster contributes to elevated triglycerides and severity of CAD by interfering with microRNA 3201 binding efficiency. J. Am. Coll. Cardiol. 64, 267-277 (2014).

27. Caussy, C. et al. An APOA5 3' UTR variant associated with plasma triglycerides triggers APOA5 downregulation by creating a functional miR-485-5p binding site. Am. J. Hum. Genet. 94, 129-134 (2014).

28. Garelnabi, M., Lor, K., Jin, J., Chai, F. \& Santanam, N. The paradox of ApoA5 modulation of triglycerides: evidence from clinical and basic research. Clin. Biochem. 46, 12-19 (2013).

29. Dron, J. S. et al. Loss-of-function CREB3L3 variants in patients with severe hypertriglyceridemia. Arterioscler Thromb. Vasc. Biol. 40, 1935-1941 (2020).

30. Nakade, Y. et al. Effect of adrenergic agonists on high-fat diet-induced hepatic steatosis in mice. Int J. Mol. Sci. 21, 9392 (2020).

31. Perez, D. M. alpha1-adrenergic receptors in neurotransmission, synaptic plasticity, and cognition. Front Pharm. 11, 581098 (2020).

32. Blue, D. R. et al. Pharmacological characteristics of Ro 115-1240, a selective a1A/ $1 \mathrm{~L}$-adrenoceptor partial agonist: a potential therapy for stress urinary incontinence. BJU Int. 93, 162-170 (2004).

33. Musselman, D. M. et al. A randomized crossover study to evaluate Ro 115-1240, a selective a1A/1L-adrenoceptor partial agonist in women with stress urinary incontinence. BJU Int. 93, 78-83 (2004).

34. Purcell, S. et al. PLINK: a tool set for whole-genome association and populationbased linkage analyses. Am. J. Hum. Genet. 81, 559-575 (2007).

35. Chou, W. C. et al. A functional variant near XCL1 gene improves breast cancer survival via promoting cancer immunity. Int J. Cancer 146, 2182-2193 (2020).

36. Pruim, R. J. et al. LocusZoom: regional visualization of genome-wide association scan results. Bioinformatics 26, 2336-2337 (2010).

37. The GTEx Consortium. The GTEx Consortium atlas of genetic regulatory effects across human tissues. Science 369, 1318-1330 (2020).

38. Ricchi, M. et al. Differential effect of oleic and palmitic acid on lipid accumulation and apoptosis in cultured hepatocytes. J. Gastroenterol. Hepatol. 24, 830-840 (2009).

39. Chou, W. C. et al. B-Myb induces APOBEC3B expression leading to somatic mutation in multiple cancers. Sci. Rep. 7, 44089 (2017).

\section{ACKNOWLEDGEMENTS}

We thank the Taiwan Biobank for providing the biological information and genotyping data for the research and Ms. Chia-Ni Hsiung and the Data Science Statistical Cooperation Center of Academia Sinica (AS-CFII-108-117) for statistical support. We thank the DNA Sequencing Core Facility in Academia Sinica (AS-CFII-108115) for sequencing service. We thank the investigators of the ENCODE and GTEx projects who have made their invaluable data available to the scientific community. This work was supported by grants from Academia Sinica and Ministry of Science and Technology, Taiwan (AS-KPQ-110-BioMed and MOST 110-0210-01-22-02).

\section{AUTHOR CONTRIBUTIONS}

W.C.C. and C.Y.S. conceived the study and wrote the manuscript. W.C.C. and W.T.C. carried out the statistic and public databases analyses, designed and performed all cell-based experiments. W.T.C. created the figures with Biorender.com. All authors read and approved the manuscript.

\section{COMPETING INTERESTS}

The authors declare no competing interests.

\section{ADDITIONAL INFORMATION}

Supplementary information The online version contains supplementary material available at https://doi.org/10.1038/s41525-021-00279-5.

Correspondence and requests for materials should be addressed to Chen-Yang Shen.

Reprints and permission information is available at http://www.nature.com/ reprints

Publisher's note Springer Nature remains neutral with regard to jurisdictional claims in published maps and institutional affiliations.

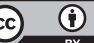

Open Access This article is licensed under a Creative Commons Attribution 4.0 International License, which permits use, sharing, adaptation, distribution and reproduction in any medium or format, as long as you give appropriate credit to the original author(s) and the source, provide a link to the Creative Commons license, and indicate if changes were made. The images or other third party material in this article are included in the article's Creative Commons license, unless indicated otherwise in a credit line to the material. If material is not included in the article's Creative Commons license and your intended use is not permitted by statutory regulation or exceeds the permitted use, you will need to obtain permission directly from the copyright holder. To view a copy of this license, visit http://creativecommons. org/licenses/by/4.0/.

(c) The Author(s) 2022 